\title{
The vertical axis wind power generation technology in Marine application feasibility analysis
}

\author{
Mofeng Qu \\ School of North China Electric Power University, Baoding 071000, China \\ 260469009@qq.com
}

Keywords: vertical axis wind turbines; classification and advantages; aerodynamic; model; the coupling; fan base.

\begin{abstract}
In this paper, I firstly expound the status of offshore wind turbine, which leads to the currently existing small vertical axis wind turbine technology gap. The following I simple introduce several common vertical axis wind turbines, their advantages and the Marine vertical axis wind turbine structure analysis of the key issues in the system, including pneumatic model, waves coupling, fan, etc. Finally, I make a summary and outlook to the offshore wind turbine construction.
\end{abstract}

\section{Introduction}

Among numerous available natural energy, wind energy is one of the world's most widely used green energy. Because of its advantages of relatively maturation compared with other new energy power, wind power generation get the favour of countries all over the world. In our country, for example, by 2014, the year adds new power generating capacity of 19.81 million $\mathrm{kW}$ wind power, which reach the historical high of power capacity. The cumulative grid installed capacity is 96.37 million $\mathrm{kW}$, accounting for $7 \%$ of the total power capacity, accounting for $27 \%$ of the world's wind power. In 2014, on-grid energy of wind power was $2014 \mathrm{~kW} \cdot \mathrm{h}$, accounting for $2.78 \%$ of the total generated energy. ${ }^{[1]}$ However, at present most wind power plant are on the land. At the same time, people have noticed restrictions of the land wind energy, such as more floor space, noise pollution problem. Because of the abundant wind energy resources and the feasibility of the current technology, ocean will become a fast developing wind power market. Affected by the relative policy (the policy of "offshore wind farm project planning outline" ${ }^{[2]}$ ), offshore wind power construction also usher in a new climax in our country. According to data, the offshore wind energy that can be the developed and utilized is about 750 million kilowatts (assuming the height is 10 meters above the sea). ${ }^{[3]}$ Since 2005, China had begun the construction of offshore wind farm, and a large number of horizontal axis wind turbine have been constructed. However, the vertical axis wind turbine was neglected. The main reason is the lacking of relevant data of the sea vertical axis wind turbine. Its development and research are in testing phase. For this purpose, aiming at problems of the vertical axis wind turbines paper, this paper makes a shallow analysis.

\section{The classification and advantages of the vertical axis wind turbines}

The vertical axis wind turbines are in variety of forms. According to the working principle of the aerodynamic, they can be divided into two kinds, resistance type and lift type. The type of Using the air resistance to the rotor blade to drive rotor rotating is called resistance type vertical axis wind turbine; The type of Using the air lift to the rotor blade to drive rotor rotating is called lift type vertical axis wind turbine.

\subsection{Resistance type vertical axis wind turbines}

Taking simple flat plate type of the vertical axis wind turbine for an example, assume wind velocity $V_{1}$ when flowing through tablet, producing an acting force $N$ to the tablet. Its direction and wind speed have the same direction. Acting force $N$ is called the resistance. If the flat area is $A$, rotating speed is $v$, to calculate the wind energy utilization coefficient: 


$$
C_{p}=\frac{P}{P_{0}}=C_{T}\left(1-\frac{v}{v_{1}}\right)^{2} \frac{v}{v_{1}}
$$

Where, $C_{T}$ is resistance coefficient of flat.

$$
\begin{gathered}
\text { When } \frac{d C_{p}}{d\left(\frac{v}{v_{1}}\right)}=0, C_{P} \text { get the maximum. Through calculating, when } \frac{v}{v_{1}}=\frac{1}{3}, C_{P} \text { get the maximum, } \\
\qquad C_{p, \max }=\frac{4}{27} C_{T}
\end{gathered}
$$

If the resistance coefficient of flat $C_{T}=1.3$, the maximum wind energy utilization coefficient of wind wheel flat is more lower than the horizontal axis wind turbines', leading to lack of competitiveness in the commercial application.

\subsection{Resistance difference type vertical axis wind turbines}

Non-flat blade type wind turbines are popular. When wind cover does not need, it can rotate at a certain speed, which is similar to the wind cup type wind meter. Windward face will change because sometimes the blades following wind and sometimes the blades are against the wind. Then the resistance coefficient will change. Wind turbines with this principle are called the resistance difference type vertical axis wind turbines. When blades follow the wind, the windward faces are spill, which has the function of gathering wind. At this moment, the wind resistance is bigger. When blades go against the wind, the windward faces are raised, the wind resistance is smaller.

The wind energy utilization coefficient is far lower than the horizontal axis wind turbines, and lack of competitiveness in the commercial application.

Set the linear velocity of blade apex is $v$, wind velocity $v_{1}$, superficial area of blade $A$, then we will know the wind energy utilization coefficient: ${ }^{[4]}$

$$
C_{p}=\frac{P}{P_{0}}=\frac{\frac{\rho}{2} A\left[C_{T 1}\left(v_{1}-v\right)^{2}-C_{T 2}\left(v_{1}+v\right)^{2}\right] v}{\frac{\rho}{2} v_{1}^{3} A}
$$

After derivation, when $\frac{d C_{p}}{d\left(\frac{v}{v_{1}}\right)}=0, \mathrm{C}_{\mathrm{P}}$ gets its maximum value. Experience shows that the wind energy utilization coefficient of wind turbines is still low, which is not often used as wind turbines.

\section{3 lift type vertical axis wind turbines}

Among Lift type vertical axis wind turbines, Darrieus type is most typical one. Meanwhile, $H$ type and $\phi$ type are most common. $\phi$ type vertical axis wind turbines are similar to the swinging jump rope. This shape can effectively reduce the effect on the blade shape of centrifugal force. However, there are certain difficulties in manufacturing, transportation and installation this special blade. In order to avoid these problems, the straight blade vertical axis wind turbines are invented, such as $H$ type.

Experimental data show that, the lift type wind turbine has higher wind energy utilization coefficient. Therefore, sea wind turbines should choose lift type wind power generator.

\section{4 vertical axis wind turbines}

Compared to the horizontal axis wind turbine, the vertical axis wind turbine has many advantages: (1) don't need wind, don't need the yaw system;(2) the effect of wind shear and the effect tower shadow is weak, small blade stress;(3) simple leaf blade structure, low manufacturing cost.(4) generator can be placed on the bottom, the stress is reasonable and easy to maintain;(5) leaf blade is multipoint beam with smaller rotating radius without cutting out;(6) wind sweeping velocity is same everywhere, the aerodynamic noise is very small;(7) wind disturbance field area is small, big power density, only 2 machine position is need between draught fan; (8) no electromagnetic pollution;(9) lightning protection;(10) generator voltage is relatively stable, which can well solve the bottlenecks in wind power development--connecting to the grid. 


\section{Pneumatic model}

Purpose of Pneumatic model research is to get the induced velocity, and ultimately determine the blade load and output power. Compared with horizontal axis wind turbine, aerodynamic model of the vertical axis wind turbine is more complex, which main contains the stream tube model, vortex model, turbulence model, dynamic stall model and computational fluid dynamics model, etc.

In the analysis, the most commonly used model is stream tube model. Stream tube model is the most commonly used vertical axis wind turbine aerodynamic model based on the momentum theory, which is equivalent to folio sine momentum model of horizontal axis wind turbine. Stream tube model is simply and can calculate the aerodynamic load impeller effectively. But shortcomings also exist: (1) under the condition of big tip speed ratio, it is difficult to calculate the induced velocity.(2) ignore the flow perpendicular to the direction of the induced velocity;(3) unable to accurately predict the impeller of the unsteady and transient load;(4) can't reflect whirlpool dropping phenomenon.

In fact, the classic aerodynamic model we often used is based on the basic assumption of uniformity. it is difficult to accurately depict the detail characteristics of flow field of vertical axis wind turbine. However, the development of computational fluid dynamics is expected to break through this bottleneck. All computational fluid mechanics models are based on the Navier - Stokes equations, which mainly include direct numerical simulation and large eddy simulation. In comparison, the large eddy simulation calculation is more accurate but more complicated. Its advantage lies in efficiency computation. But the calculation cost is still very high, which cannot be applied to practice yet.

\section{The wave coupling}

The wave coupling has been the difficulties of ocean engineering research. Multivariate extreme value theory is the theory that studies multiple related variable extreme, which discusses the multiple related variable extremer of joint distribution method. This theory is the effective measure to consider all sorts of extreme load combination. It won a wide application in ocean engineering. However, related structure analysis in multivariate extreme value distribution is very complex, and solving the function also is invisible extreme function more, by the higher mathematics is the only through iteration. Once the variable complex calculations will be very trouble, which is not conducive to the practical application.

The above value theory is based on the extreme of single load. At last, through several combination coefficients or some kind of complex combination, we get extreme value of static analysis for under the action of wind, which is the result of static analysis in essence. Because we only do static analyses, the extreme value of each load usually do not appear at the same time. Therefore, they cannot be simply edited, leading to load combination of various theories or methods. In fact, load and load effect are nonlinear and they have no simple superposition character. So one-to-one corresponding relation cannot be established between maximum load combination and the most unfavorable effect. Therefore, the above combinations are not applicable in the case of considering nonlinear.

According to analysis above, we found that through the static analysis under the premise of complex iterative, waves coupling results of the multivariate extreme value theory can be calculated. However, it is defective and not practical, which could not be directly taken into the practical construction. And more improvement is still need in the future.

\section{The fan foundation}

The fan foundation can be divided into land fan foundation and sea fan foundation. Due to changes of environmental conditions, there are essential difference between land fan basis and sea fan basis. Sea fan location can be divided into tidal flats, offshore and deep sea. According to the difference of geological conditions, the offshore foundation forms mainly include gravity fan basis, negative pressure bucket foundation, pile foundation, spider foundation, jacket foundation and elevated pile 
caps foundation. Deep-water fan foundation is in suspension, and tidal flats fan base is usually between land fan foundation and sea fan foundation.

As we all know, whether the fan foundation is firm, and how to selection construction determines the fan in the area, as for the selection of the fan foundation, there are still many problems: (1) soil power weakening effect. Soil power weakening effect refers to the decreasing of soil stiffness around the pile under the increasing of cycling times. (2) The effect of soil plug. in the process of Steel pipe piles, the soil plug into steel pipe forming soil plug, by changing the end resistance and side resistance to achieve the improvement of pile bearing capacity, this is called the effect of soil plug.(3) waves induced seabed instability. Seabed instability is a special kind of offshore wind turbine foundation failure mode.it mainly caused by fluctuations in the seabed. Many experts and scholars have made a lot of research about the above problems, but there is still no accurate method that can be applied to the actual construction. Therefore, the key to solve these problems is to feasibility of Marine vertical axis wind turbine construction is to find a better way.

\section{Summary}

This article mainly aims at the common problems in Marine vertical axis wind turbine. Through access to relevant data, combined with knowledge of vertical axis wind turbine, shallow analysis has been made. Conclusions as follow are got:

Wind energy utilization coefficient of lift type vertical axis wind turbine is higher. And it has many virtues. So it shall be considered as the offshore wind power generators.

Rotor aerodynamic model is relatively mature. But the classical aerodynamic model is under the premise of the assumptions, which is unable to meet the actual situation of complex; Computational fluid dynamics model of computer computing power demand is higher. It is not suitable for practical engineering and more research is need.

Wave coupling has always been bothering people of focusing on the study of Marine engineering problem. At present, the most advanced multivariate extreme value theory is expounded on the basis of the principle of statics, which could not solve this problem yet. So the difficulty hasn't been overcome.

Fan foundation is the basis of wind turbine parts and the key to keep the wind turbine safe and efficient. However, influenced by ocean actual situation, it is not a perfect method suitable for all kinds of actual situation. Until now, there are no thorough methods suitable for all kinds of situation. Therefore, it needs long-term on-the-spot investigation and argumentation to build the fan foundation.

In general, all countries are still in research stage when it comes to applying the vertical axis wind turbines offshore. Many big problems that easy to detect haven't been solved. But, vertical axis wind turbine has many advantages compared to horizontal axis wind turbines because the vast sea area and rich wind. So it is more suitable for application in maritime. It is true that sea vertical axis wind turbine has a broad application prospects, but for its further research in the future will be a long process. At last, we hope that above analysis could do something to the construction of vertical axis wind turbine and filling a blank in the vertical axis wind turbine technology.

\section{References}

[1] National energy administration: 2014 adds new power generating capacity of 19.81 million mw wind record [online] http://money.163.com/15/0212/15/AI8UQGLT00253B0H.html

[2] Offshore wind farm project planning outline [online] http:// wenku.baidu.com/ view/ a9af8c 68a45 177232f60a283.html

[3] Mr.li. Wind 12 in China [M]. Beijing: chemical industry press. 2005 (84): 106107.

[4] He Jie, Yang xin, Yang jiasheng. The main influence factors analysis of offshore wind farm planning [J]. Journal of Chinese subject learning. 2010 (11) : 17, 18 
[5] Liu qi, Xuyiqing. Several issues a preliminary study of the offshore wind power development in our country [J]. Journal of Shanghai electric power, 2007 (2) : 144148

[6] Milne - Thomson L m. heoretical aerodynamics [M]. London: Macmillan, 1952 (5): 40, 60. 